\title{
Article \\ Small-Signal Analysis of All-Si Microring Resonator Photodiode
}

\author{
Yiwei Peng, Wayne V. Sorin (1), Stanley Cheung, Yuan Yuan *(i), Zhihong Huang, Marco Fiorentino \\ and Raymond G. Beausoleil
}

Hewlett Packard Labs, Hewlett Packard Enterprise, Milpitas, CA 95035, USA; yiwei.peng@hpe.com (Y.P.); wayne.sorin@hpe.com (W.V.S.); stanley.cheung@hpe.com (S.C.); zhihong.huang@hpe.com (Z.H.); marco.fiorentino@hpe.com (M.F.); ray.beausoleil@hpe.com (R.G.B.)

* Correspondence: yuan.yuan@hpe.com

\begin{abstract}
All-silicon microring resonator photodiodes are attractive for silicon photonics integrated circuits due to their compactness, wavelength division multiplexing ability, and the absence of germanium growth. To analyze and evaluate the performance of the microring photodiode, we derived closed-form expression of the response transfer function with both electrical and optical behavior included, using a small-signal analysis. The thermo-optic nonlinearity resulting from optical loss and ohmic heating was simulated and considered in the model. The predicted response achieved close agreement with the experiment results, which provides an intuitive understanding of device performance. We analytically investigated the responsivity-bandwidth product and demonstrated that the performance is superior when the detuning frequency is zero.
\end{abstract}

Keywords: photodiodes; resonators; photothermal effects; silicon; optoelectronics

check for updates

Citation: Peng, Y.; Sorin, W.V.; Cheung, S.; Yuan, Y.; Huang, Z.; Fiorentino, M.; Beausoleil, R.G. Small-Signal Analysis of All-Si Microring Resonator Photodiode. Electronics 2022, 11, 183. https:// doi.org/10.3390/electronics11020183

Academic Editor: Dongseok Suh

Received: 30 November 2021

Accepted: 5 January 2022

Published: 7 January 2022

Publisher's Note: MDPI stays neutral with regard to jurisdictional claims in published maps and institutional affiliations.

Copyright: (c) 2022 by the authors. Licensee MDPI, Basel, Switzerland. This article is an open access article distributed under the terms and conditions of the Creative Commons Attribution (CC BY) license (https:// creativecommons.org/licenses/by/ $4.0 /)$.

\section{Introduction}

The silicon photonics platform has been considered the most attractive solution for high-speed network and artificial intelligence applications due to its low cost and high miniaturization $[1,2]$. Because the cutoff wavelength of bulk Si is only around $1.1 \mu \mathrm{m}$, the photodetection at telecommunication wavelengths is usually achieved by the heteroepitaxial growth of germanium (Ge) or heterogeneous integration of III-V materials [3-6]. Both methods require added material costs and extra process steps. All-Si photodetection based on defect state absorption has been demonstrated to achieve high quantum efficiency and high bandwidth [7,8]. However, the method needs special steps, such as defects implantation, which are not compatible with the standard process in foundries. Si photodetectors (PDs) based on two-photon absorption (TPA) and photon-assisted tunnelling (PAT) can absorb sub-bandgap wavelengths [9]. Applying a high bias voltage across the PN junction, the spatial distance between the conduction and valence band will be shortened due to the strong electric field. The electrons in the valence band can possibly tunnel into the conduction band [10]. However, the mechanisms are weak and PDs suffer from low responsivity. Microring resonator (MRR) PD benefits from optical resonant enhancement effect is a promising solution to improve responsivity [11]. Intel has reported a $112 \mathrm{~Gb} / \mathrm{s}$ (56 GBaud PAM4) all-Si MRR PD with a responsivity of $0.23 \mathrm{~A} / \mathrm{W}$ [12].

Thus far, there is not much dynamic analysis work to quantify the performance of the MRR PD. Some steady-state and dynamical discussions on MRR modulators show that the modulation rate and efficiency are limited by the resonator quality factor (Q) [13-18]. For the MRR PDs, $Q$ value limits the rate at which the optical power can be injected in the ring and absorbed, which determines the modulation speed. At the same time, the resonant enhancement effect, and thus the responsivity, are related to the $Q$ value. Bandwidth improvement always comes at the expense of a reduced responsivity and it is important to weigh the tradeoff in design [19]. The performance of MRR PD also 
depends on the input wavelength offset away from the resonance wavelength. Optical power losses and electrical power consumption within the small volume usually lead to heat accumulation as well as thermally-induced nonlinearities, which make the model more complicated because thermal power distribution in MRR can dynamically change the resonance wavelength [20-23]. For high-power applications, the thermal nonlinearity of the MRR PD cannot be ignored. To completely understand the performance of MRR PD, a fully dynamical model considering thermal nonlinear effects is required.

In this paper, we provide a small-signal analysis of all-Si MRR PD in terms of frequency response and responsivity. The perturbation treatment is applied to derive the expression of the response based on the MRR energy coupling model. We take thermal nonlinear effects into consideration by simulating the temperature variance and calculating the temperature induced resonance shift. The results predicted from the model match well with the measured data for our high-performance Ge-free MRR PD with the simple Si PN junction. The responsivity-bandwidth, which is an important parameter for PD [24,25], is also analyzed versus the detuning frequency. The verified model helps us understand the MRR PD and is appealing to future all-Si PD optimization.

\section{Analytic Model}

In this section, we aim to derive the small-signal response of the MRR PD. As shown in Figure 1, the transfer function of MRR PD can be divided into optical response $H_{O}\left(\omega_{m}\right)=\frac{P_{r}}{P_{i n}}$ and electrical response $H_{E}\left(\omega_{m}\right)=\frac{I_{L}}{I_{s}}$, where $P_{r}$ and $P_{i n}$ are optical power in cavity and input optical power and $I_{L}$ and $I_{S}$ are load current and source current, respectively. $P_{r}$ is related to $I_{s}$ by $P_{r}=\frac{I_{s}}{R}$, where $R$ is the responsivity of the MRR at critical coupling at resonant wavelength. The input optical signal consists of a small sinusoidal signal of $\cos \left(\omega_{m} t\right)$ and an optical carrier of $\cos \left(\omega_{i n} t\right)$, where $\omega_{m}$ and $\omega_{\text {in }}$ are modulation angular frequency and optical carrier frequency. Under stimulus, the RF part of the output signal oscillates at $\omega_{m}$.

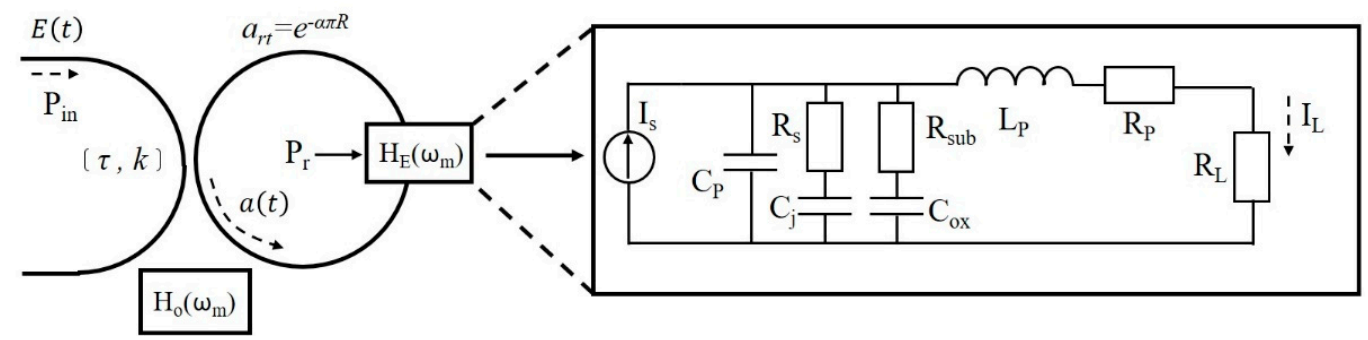

Figure 1. MRR PD response block diagram.

In order to obtain the optical response of the PD, we started with the rate equations as [26-28]:

$$
\frac{d a(t)}{d t}=\left(j \omega_{r}-\gamma_{r}\right) a(t)-j \mu E(t)-j \mu \delta E(t)
$$

where $a(t)$ is the energy amplitude travelling inside the resonator, $\omega_{r}$ is the angular resonant frequency of the ring, $E(t)$ is the input amplitude of waveguide mode and $\delta E(t)$ is the modulated amplitude. $\gamma_{r}$ is the amplitude decay rate which consists of a decay rate due to the attenuation in the resonator $\gamma_{l}$ and the ring to bus waveguide coupling $\gamma_{e}$. The $Q$ factor and photon lifetime $\tau_{p}$ can be obtained by $Q=\frac{2 \gamma_{r}}{\omega_{r}}=\frac{1}{\omega_{r} \tau_{p}}$. The energy coupling coefficient $\mu$ is related to the decay rate $\gamma_{e}$ and field coupling coefficient $k$ by $\mu^{2}=2 \gamma_{e}=\frac{k^{2}}{T_{r t}}$, where $T_{r t}$ is the round-trip transit time. For the steady state, the input field is written as $E(t)=E_{0} e^{j \omega_{i n} t}$ and the energy amplitude is $a(t)=a_{0} e^{j \omega_{i n} t}$, where $a_{0}$ is:

$$
a_{0}=\frac{-j \mu E_{0}}{j \Delta \omega+\gamma_{r}}
$$


$\Delta \omega=\omega_{\text {in }}-\omega_{r}=2 \pi \Delta f$ is the angular frequency detuning of the input carrier frequency from the resonance frequency.

With the sinusoidal modulated signal, the energy amplitude will experience a small amount of change $\delta a(t)$. To solve the equation, the $\delta a(t)$ is assumed to be the product of a slowly varying envelope and an exponential item: $\delta a(t)=\delta a e^{\left(j \omega_{r}-\gamma_{r}\right) t}$. The modulated signal can be written with upper and lower sidebands as: $\delta E(t)=0.5 \times$ $\delta E_{0}\left(e^{j\left(\omega_{i n}+\omega_{m}\right) t}+e^{j\left(\omega_{i n}-\omega_{m}\right) t}\right)$. The output signal consists of beat signals generated between the carrier and sidebands and between two sidebands. Because the modulation depth is small, the high order harmonics can be neglected. The $\delta a(t)$ can be written as:

$$
\begin{array}{r}
\delta a(t)=-0.5 \times j \mu \delta E_{0}\left(\frac{1}{j \Delta \omega+j \omega_{m}+\gamma_{r}} e^{j\left(\omega_{i n}+\omega_{m}\right) t}\right. \\
\left.+\frac{1}{j \Delta \omega-j \omega_{m}+\gamma_{r}} e^{j\left(\omega_{i n}-\omega_{m}\right) t}\right)
\end{array}
$$

The output signal depends on the absorption coefficient due to PAT and optical power in the resonator. The transfer function can be written as: $H_{O}\left(\omega_{m}\right)=\frac{1}{E_{0}{ }^{2} T_{r t}}|(a(t)+\delta a(t))|^{2}$. The transfer function can be divided as the DC part: $\frac{1}{E_{0}{ }^{2} T_{r t}}|a(t)|^{2}$ and RF part: $\frac{1}{E_{0}{ }^{2} T_{r t}}\left(a(t) \delta a^{*}(t)+a^{*}(t) \delta a(t)\right)$. By substituting Equations (2) and (3), the DC and RF part of $H_{O}\left(\omega_{m}\right)$ can be written as:

$$
\begin{gathered}
H_{O-D C}=\frac{1}{T_{r t}} \frac{\mu^{2}}{\Delta \omega^{2}+\gamma_{r}{ }^{2}} \\
H_{O-A C}\left(\omega_{m}\right)=\frac{1}{T_{r t} E_{0}} \frac{2 \gamma_{r} \delta E_{0} \mu^{2}}{\Delta \omega^{2}+\gamma_{r}^{2}}\left[\frac{j \omega_{m}+\frac{\Delta \omega^{2}+\gamma_{r}{ }^{2}}{\gamma_{r}}}{\left(j \omega_{m}+\varepsilon\right)\left(j \omega_{m}+\varepsilon^{*}\right)}\right]
\end{gathered}
$$

where $\varepsilon=j \Delta \omega+\gamma_{r}$.

The equivalent circuit of PD is shown in Figure 1. $C_{j}$ and $R_{S}$ represent series capacitance and resistance from the Si PN junction. $C_{o x}$ and $R_{s u b}$ represent the capacitance from oxide layer and resistance from the high-resistivity substrate. $C_{P}, R_{P}$, and $L_{P}$ are parasitic components. All the parameters can be extracted from the S11 fitting and the electrical response can be modeled with equivalent resistance $R_{\ell q}$ and capacitance $C_{\ell q}$ as:

$$
H_{E}\left(\omega_{m}\right)=\frac{1}{1-\omega_{m}^{2} L_{P} C_{e q}+j \omega_{m} C_{e q} R_{e q}}
$$

The small-signal transfer function of the MRR PD is the product of $H_{E}\left(\omega_{m}\right), H_{O}\left(\omega_{m}\right)$, and $R$. The MRR PD is a fourth-order system with a complex-conjugate pole pair, two poles from the RLC circuit and a zero. The pole pair and zero depend on the $Q$ value and laser detuning frequency, which determine the speed of the PD. The product of $H_{O-D C}$ and $R$ is the responsivity of the MRR PD.

The responsivity-bandwidth product, which is defined as the product of the $3 \mathrm{~dB}$ bandwidth and the responsivity, is an important figure of merit to characterize the performance of the PD. Figure 2 shows the relationship between the responsivity-bandwidth product versus the detuning frequency and decay rate. During the calculation, the responsivity was normalized as $1 \mathrm{~A} / \mathrm{W}$ when $\gamma_{r}=2 \pi \times 10 G$ at zero detuning. The detailed normalized responsivity and bandwidth at zero detuning are shown as star markers in the Figure 2. It can be observed that the responsivity-bandwidth product decreases with the laser detuning frequency for a fixed decay rate. Therefore, the PD can achieve the best performance when the optical carrier frequency is set as the same as the resonance frequency. When $\Delta \omega=0$, the $3 \mathrm{~dB}$ bandwidth for MRR equals to $\frac{\gamma_{r}}{2 \pi}$ if we neglect the RC time limitation. It should be noted that critical coupled resonator is favorable for the MRR PD responsivity only if the PAT effect is much higher than the loss or is proportional to the power decay rate in cavity. The experimental relationship between frequency response and detuning frequency will be discussed in the following section. The bandwidth can be improved with a higher decay 
rate at a cost of lower responsivity. To obtain the high output signal, the MRR PD usually needs to work at a high optical power density. The heating power will accumulate in the small volume, which will increase the temperature of the structure and shift the resonance frequency [20]. To analyze the PD based on Equations (4) and (5), the thermal-induced shift needs to be calculated.

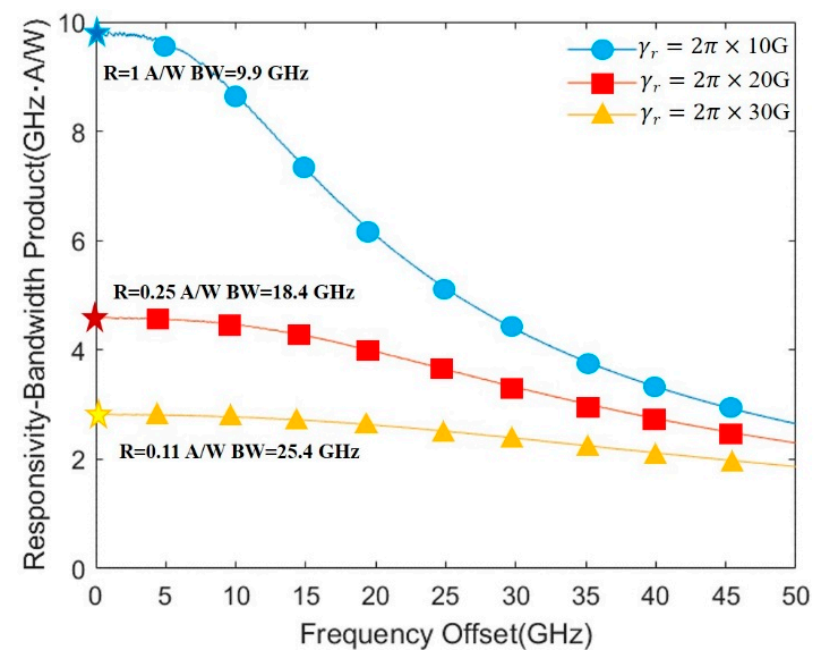

Figure 2. Calculated responsivity-bandwidth product of a MRR PD for several values of the amplitude decay rate $\gamma_{r}$.

The optical energy in the MRR can be dissipated due to mode mismatch, propagation loss, bend loss and free-carrier absorption (FCA) [29]. For the doped Si MRR, the FCA usually dominates the loss $[30,31]$. The heating power resulted from the optical loss at low bias voltage can be described as:

$$
P_{o p t}=\alpha_{F C A}|a(t)|^{2} \approx\left[1-\left(a_{r t}\right)^{2}\right] \times|a(t)|^{2}
$$

where $\alpha_{F C A}$ is the loss rate due to FCA and $a_{r t}$ is the round-trip field attenuation factor, which can be obtained from the intrinsic loss induced decay rate $a_{r t}=e^{-\gamma_{l} T_{r t}}$.

Another dissipated power source is the ohmic heating, which can be determined by [32]:

$$
P_{\text {elec }}=P_{D C, \text { in }}-P_{R F, \text { out }}
$$

$P_{D C \text {, in }}$ represents the DC electrical power delivered to the PD and $P_{R F, \text { out }}$ represents the RF power delivered to the load. $P_{D C, \text { in }}$ is approximately equal to the product of the current and applied voltage. Because the voltage is mainly applied at the PN junction in the waveguide, the heat will accumulate in this area, where it largely overlaps with the mode area. Due to limited thermal conductivity, the heat will change the temperature of the structure and consequently change the refractive index as well as the resonance wavelength. The change in resonant wavelength as a function of the waveguide temperature variation can be expressed as [33,34]:

$$
\Delta \lambda_{r}(\Delta T)=\lambda_{r}\left[1+\left(\in+\frac{1}{n_{g}} \frac{d n}{d T}\right) \Delta T\right]
$$

where $\in$ and $n_{g}$ are the thermal expansion coefficient and group index of the MRR. $\frac{d n}{d T}$ is the index thermo-optic coefficient of silicon, which is $\sim 1.94 \times 10^{-4} \mathrm{~K}^{-1}$ at $1310 \mathrm{~nm}$.

To analyze the small-signal response, including the nonlinear thermal optical effect of a MRR PD, we first need to obtain the static optical power in the MRR by calculating the resonator optical enhancement. The heating power and generated temperature variation can then be used to calculate the resonator frequency shift as well as the detuning frequency. Finally, Equations (5) and (6) will tell us the frequency response. 


\section{Experimental Verification}

To verify the small-signal model in the previous section, we designed an all-Si MRR PD at Advanced Micro Foundry (AMF). The cross section of the MRR PD and a microscope image of the fabricated device are shown in Figure 3a,b. The investigated PD has an equivalent radius of $12 \mu \mathrm{m}$ and was implemented on a $220 \mathrm{~nm}$ SOI wafer with a remaining slab height of $90 \mathrm{~nm}$. A simple PN junction was formed within the $500 \mathrm{~nm}$ wide waveguide.

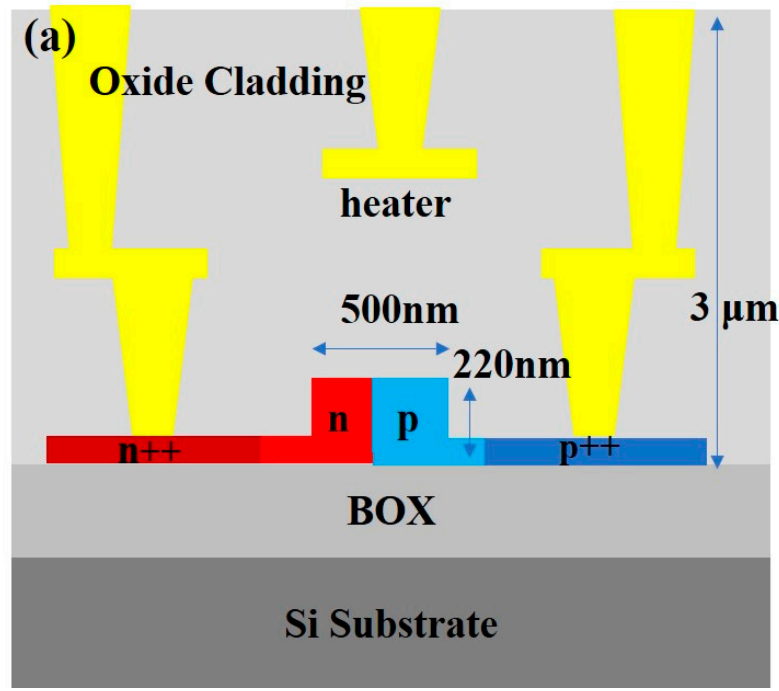

(b)

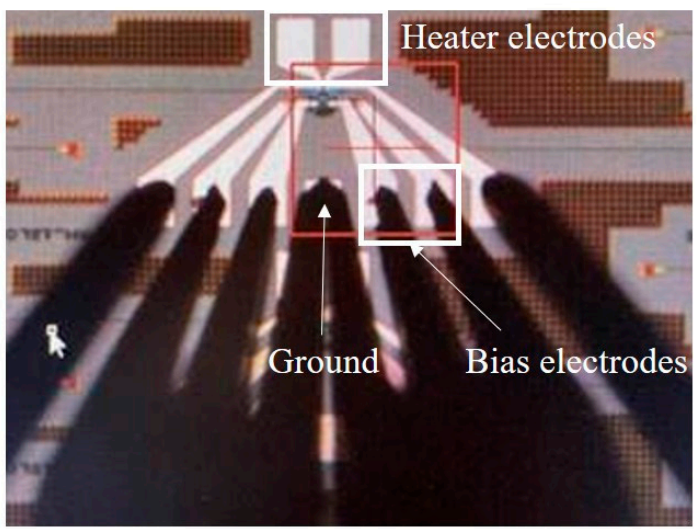

Figure 3. (a) Cross section of the MRR waveguide in the active region; (b) microscope image of a MRR PD.

\subsection{Response}

Figure 4a shows the measured transmission spectrum of the MRR around $1310 \mathrm{~nm}$ with low optical input power $(-4.5 \mathrm{dBm})$ when the bias voltage is $-6 \mathrm{~V}$. The extinction ratio of the resonator is $\sim 10 \mathrm{~dB}$ and $\mathrm{Q}$ factor is $\sim 7000$. The field coupling coefficient $k$ and the round-trip field attenuation factor $a_{r t}$ can be extracted as 0.36 and 0.96 , respectively, by fitting the measured spectrum using the equations as shown in [28]. The attenuation mainly comes from the high impurity concentration in the MRR waveguide area and PAT effect.
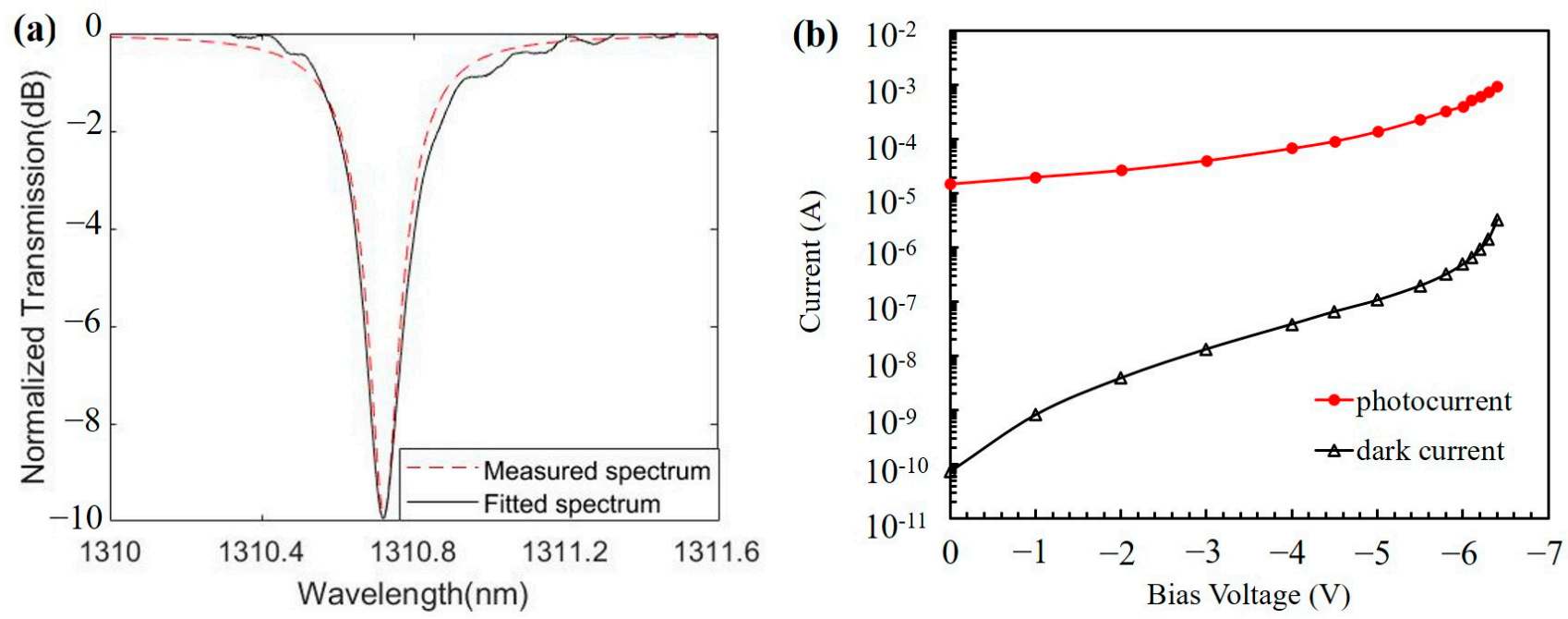

Figure 4. (a) Measured and fitted transmission spectrum with bus waveguide power of $-4.5 \mathrm{dBm}$; (b) Measured photo and dark current with bus waveguide power of $6.5 \mathrm{dBm}$. 
The measured photo and dark current are demonstrated in Figure $4 \mathrm{~b}$. The current was measured at its highest point $(\sim 1311.6 \mathrm{~nm})$ when the optical input power is $6.5 \mathrm{dBm}$. The current increases with the reverse bias due to the enhanced PAT effect and the avalanche gain. At $-6 \mathrm{~V}$, the MRR PD shows a dark current of $0.5 \mu \mathrm{A}$ and a photo current of $0.41 \mathrm{~mA}$.

\subsection{Thermal-Induced Nonlinearity}

For the MRR PD, the CW transmission spectrum can be significantly modified with thermal-induced nonlinearity. The optical transmission spectrum with high optical input power $(6.5 \mathrm{dBm})$ was measured at $-6 \mathrm{~V}$ and is shown in Figure 5 . We observe the resonance wavelength redshifts until the wavelength scans up to $\sim 1311.6 \mathrm{~nm}$. The nonlinear refractive index change can be attributed to the increase of temperature resulting from the ohmic heating and optical absorption. Beyond this wavelength, the heating power disappears, and the resonance shifts back to the cold cavity value.

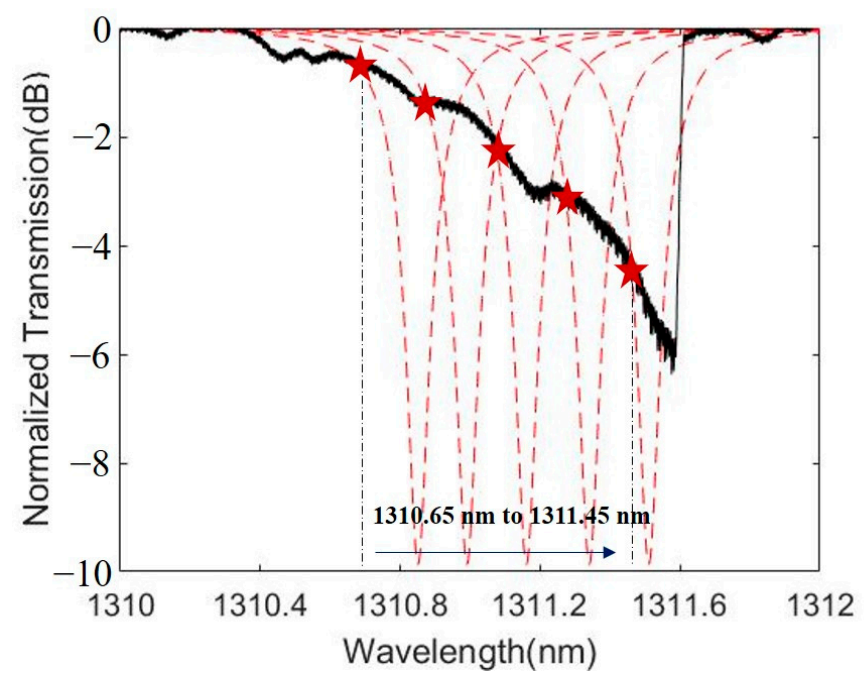

Figure 5. Transmission spectrum with bus waveguide power at $6.5 \mathrm{dBm}$.

In order to calculate the small-signal response at different input wavelengths, the nonlinear resonance shift and detuning frequency are required. The easiest way to obtain these is based on the transmission power given by the spectrum. Because the nonlinear loss from TPA is small, we can assume that the loss coefficient does not change significantly with the optical power [22,35]. The calculated linear spectrums at different input wavelengths are shown as dotted lines in Figure 5. The difference between input and resonance wavelength can be calculated as the transmission power $T_{a p}$ and is known using the following formula [28]:

$$
T_{a p}\left(\varnothing_{r t}\right)=\frac{\tau^{2}+a_{r t}^{2}-2 \tau a_{r t} \cos \varnothing_{r t}}{1+\tau^{2} a_{r t}^{2}-2 \tau a_{r t} \cos \varnothing_{r t}}
$$

where $\tau$ is the field transmission coefficient $\left(\tau=\sqrt{1-k^{2}}\right)$ and $\varnothing_{r t}$ is the round-trip phase, which is related to the detuning frequency. The calculated resonances at different input wavelengths are shown in Table 1.

To verify the above analysis, Lumerical HEAT was used to simulate temperature variation when the bus waveguide power is $6.5 \mathrm{dBm}$ and bias voltage is $-6 \mathrm{~V}$. The input power consists of the ohmic heating and optical loss. Ohmic heating is proportional to the product $I_{\text {total }} V_{\text {bias }}$, which will accumulate around the junction. Total optical attenuation can be divided into absorption due to PAT that generates photocurrent and loss mainly due to FCA that generates heat. The power loss coefficient, which is about $6.5 \%$ per round trip, can be extracted from the transmission spectrum at low bias when the PAT effect is negligible. The detailed simulation parameters are shown in the Table 1. 
Table 1. Simulated and calculated resonance shift at different input wavelengths.

\begin{tabular}{cccccc}
\hline$\lambda$ & $\mathbf{1 3 1 1 . 4 5}$ & $\mathbf{1 3 1 1 . 2 5}$ & $\mathbf{1 3 1 1 . 0 5}$ & $\mathbf{1 3 1 0 . 8 5}$ & $\mathbf{1 3 1 0 . 6 5}$ \\
\hline Ohmic heating $(\mathrm{mW})$ & 1.85 & 1.54 & 1.12 & 0.84 & 0.52 \\
Optical loss $(\mathrm{mW})$ & 1.65 & 1.37 & 1 & 0.75 & 0.46 \\
Simulated $\Delta T(\mathrm{~K})$ & 12.1 & 9.8 & 7.1 & 5.2 & 3.1 \\
Simulated $\Delta \lambda_{\text {res }}(\mathrm{nm})$ & 0.78 & 0.63 & 0.46 & 0.33 & 0.2 \\
Calculated $\Delta \lambda_{\text {res }}(\mathrm{nm})$ & 0.8 & 0.64 & 0.47 & 0.33 & 0.19 \\
Detuning $\Delta f(\mathrm{GHz})$ & 11.1 & 14.6 & 20.1 & 25.5 & 35.2 \\
\hline
\end{tabular}

The simulated temperature variation at $1311.45 \mathrm{~nm}$ is shown in Figure 6. The temperature at the bottom of the simulation region (in the Si substrate) was set to the room temperature of $300 \mathrm{~K}$. It can be observed that there is a strong localization of temperature variation near the waveguide area. The average temperature rises by $12.1 \mathrm{~K}$ and the corresponding resonance shift is $0.78 \mathrm{~nm}$. We observe close agreement with the calculated value based on the transmission spectrum, which corroborates the thermal analysis. The temperature variation and induced resonant shift at other wavelengths were also simulated and are shown in Table 1. By linear fitting, the thermal impedance of the structure is $\sim 3.45 \mathrm{~K} / \mathrm{mW}$.

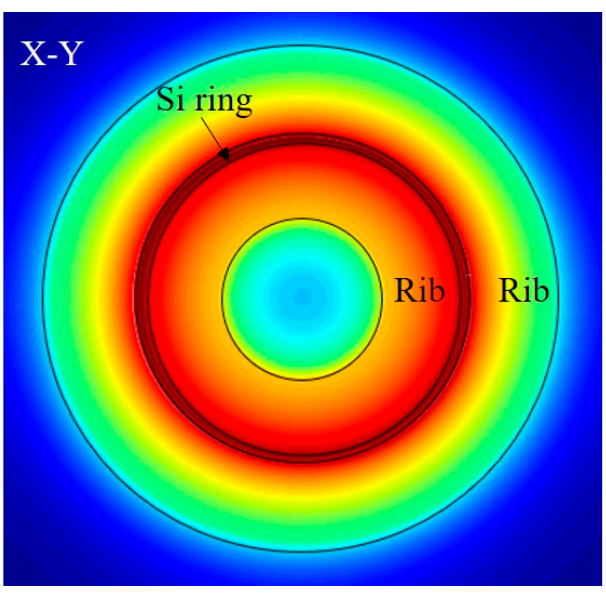

(a)

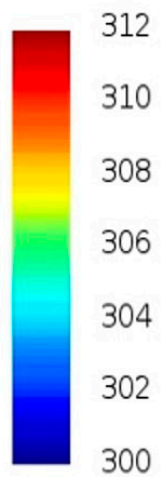

$\Delta \mathrm{T}($ Kelvin $)$

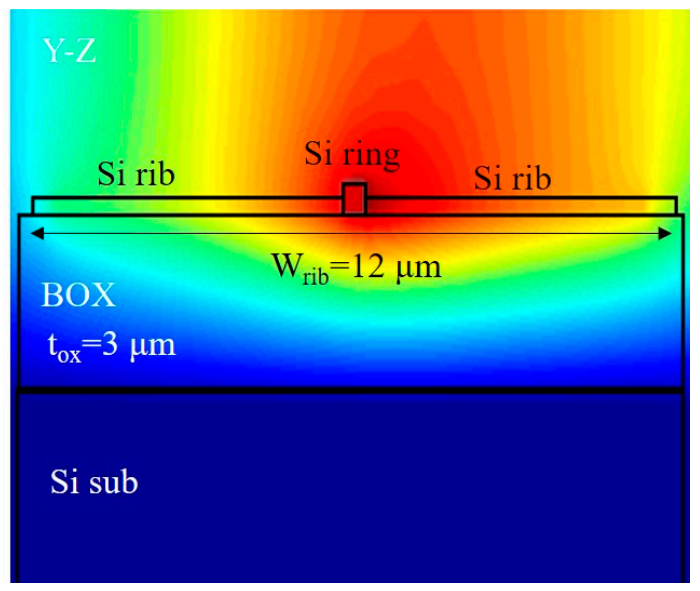

(b)

Figure 6. Simulated temperature variation when the input wavelength is $1311.45 \mathrm{~nm}$ in (a) X-Y plane and (b) Y-Z plane.

The thermal impedance was also measured when the input optical power is $-4.5 \mathrm{dBm}$ and the thermal nonlinear effect can be neglected. As shown in Figure 7, the measured resonance shift versus heat power was $215 \mathrm{pm} / \mathrm{mW}$ and the corresponding thermal impedance was $3.37 \mathrm{~K} / \mathrm{mW}$, which is close to the simulated value. The small discrepancy may come from the small piece of contact metal on the rim that was not included in the simulation. The relatively high thermal impedance can be attributed to the poor thermal conductivity of $\mathrm{SiO}_{2}$ and thick BOX.

The nonlinear coefficient $n_{T O}$, due to the thermo-optic effect, can be defined as $n_{T O}=\frac{\Delta n \times A_{e f f}}{P_{r}}$, where $\Delta n$ is the nonlinear index change and $P_{r}$ is the optical power in the MRR. $A_{e f f}$ is the effective mode area, which was simulated in Lumerical MODE as $0.15 \mu \mathrm{m}^{2}$. The calculated nonlinear coefficient is $0.15 \mathrm{~cm}^{2} / \mathrm{GW}$, which is comparable to the results in [20]. The value is more than 3 orders of magnitude higher compared to the Kerr effect in $\mathrm{Si}$, which can be attributed to the photocurrent generated in the MRR PD [36].

\subsection{Frequency Response}

In the small-signal equivalent circuit model as shown in Figure 1, the series capacitance $C_{j}$, oxide layer capacitance $C_{o x}$, and parasitic capacitance $C_{P}$ are $5.7 \mathrm{fF}, 5.8 \mathrm{fF}$, and $36.8 \mathrm{fF}$, 
respectively. The series resistance $R_{s}$, substrate resistance $R_{s u b}$, and parasitic resistance $R_{P}$ are $91 \Omega, 1967 \Omega$, and $22 \Omega$, respectively. Parasitic inductance $L_{P}$ is $40 \mathrm{pH}$. All the components are extracted from $S$ parameter fitting. The load resistance $R_{L}$ is $50 \Omega$. Based on these values, the RLC-limited $3 \mathrm{~dB}$ bandwidth of the MRR PD is $~ 66 \mathrm{GHz}$.

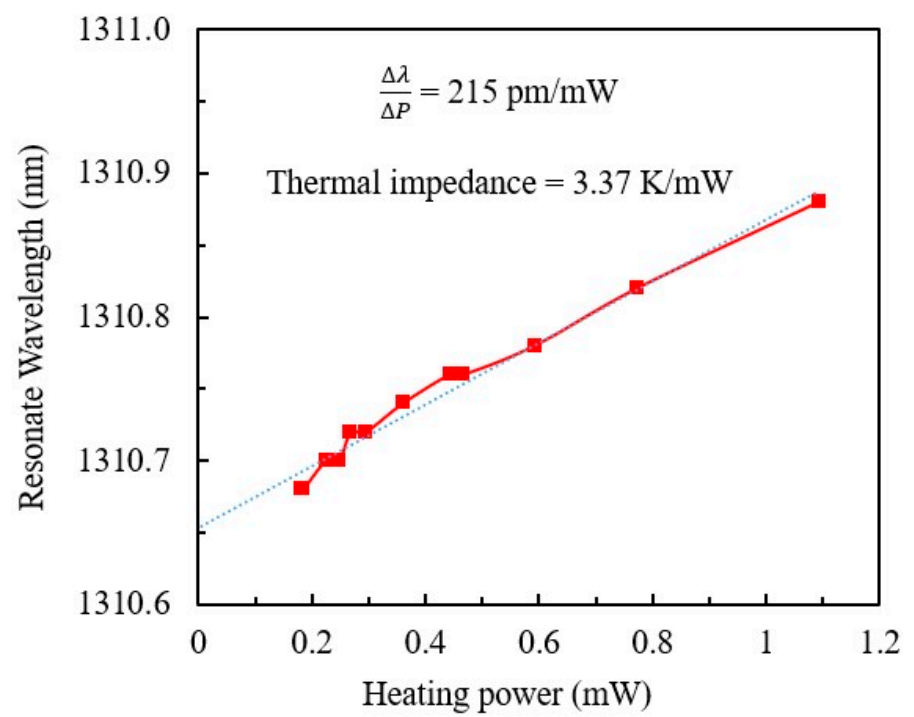

Figure 7. Measured thermal impedance of the MRR PD.

Substituting the laser detuning obtained in the last subsection and other MRR parameters in Equation (5), the frequency responses of the MRR PD are calculated and shown as the solid lines in Figure 8. The S21 were measured for input wavelength from $1310.65 \mathrm{~nm}$ to $1311.45 \mathrm{~nm}$ at $-6 \mathrm{~V}$ with a commercial $65 \mathrm{GHz} \mathrm{MZM}$ and a vector network analyzer (VNA). The measurement results are also shown in Figure 8 with dotted lines, and they show excellent agreement with our calculated results. The data is normalized to the DC value at $1311.45 \mathrm{~nm}$. The small ripples in the measured response are due to the multiple reflections of the signal at the GSG probe and the high-speed electrical lines. It can be observed that peaking occurs when the detuning frequency is large. This can be explained by the fact that the absolute value of the zero in the transfer function becomes larger than the pole pair. Although the bandwidth can be improved with detuning frequency, the output signal at high frequency is weaker and the responsivity-bandwidth product decreases as shown in Figure 2. It can be concluded that the best operating wavelength for MRR PD is the resonance wavelength to obtain the maximum output power at each frequency. When the detuning frequency is zero, the $3 \mathrm{~dB}$ bandwidth of the MRR PD can be simplified as $\sqrt{\frac{1}{\left(\frac{2 \pi}{\gamma_{r}}\right)^{2}+\left(\frac{1}{f_{R L C}}\right)^{2}}}$.

The MRR PD eye diagrams were measured and are shown in Appendix A. Our PDs can support $80 \mathrm{~Gb}$ /s nonreturn-to-zero (NRZ) signal and $100 \mathrm{~Gb} / \mathrm{s}$ PAM4 signal transmission. The summary of the all-Si PDs and APDs is shown in Appendix B. 


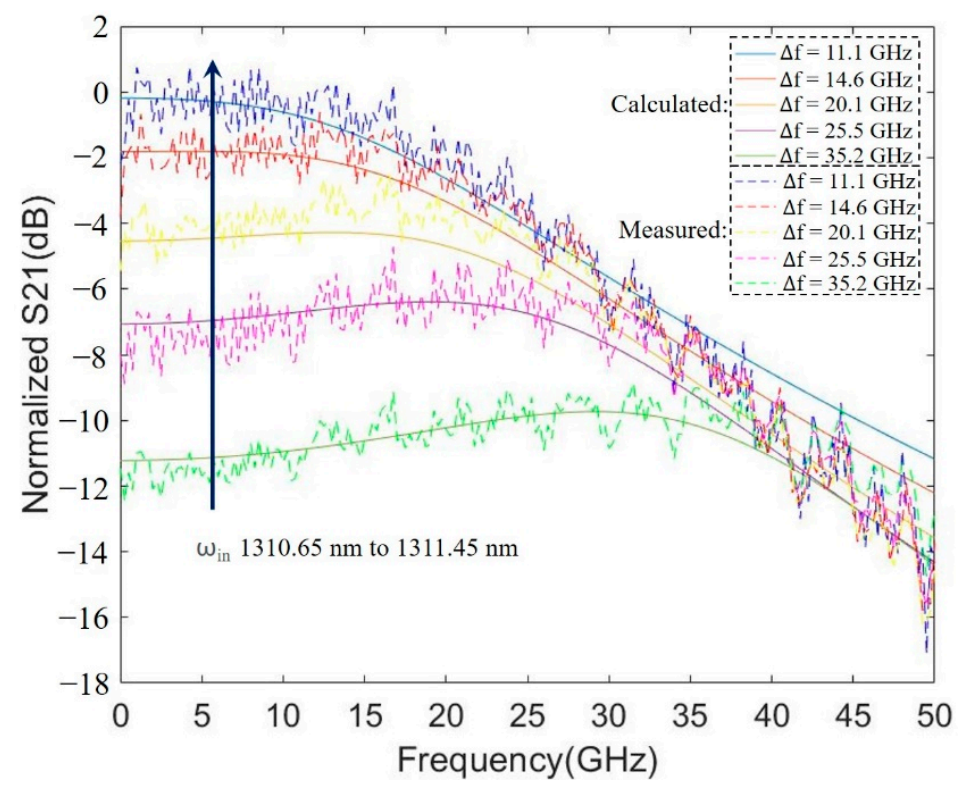

Figure 8. Measured and simulated frequency response at different input wavelength.

\section{Discussion and Conclusions}

For the first time, we modeled the small-signal response of MRR PDs that can predict the frequency response as well as the responsivity. We derived the thermal- and frequencydependent transfer function based on the rate equation and perturbation theory. Both electrical and optical behavior of the PD are considered in the expression, which suggests that a MRR PD is a fourth-order system with one zero, a pair of complex-conjugate poles and two poles from the RLC circuit. The thermo-optic nonlinear effect was investigated, and the simulated thermal impedance has been verified with measurement. The model has been verified experimentally to be accurate and allows for the determination of an $\mathrm{O} / \mathrm{E}$ response of a MRR PD with high precision. The responsivity-bandwidth product is adopted as a figure of merit to demonstrate the performance of PD is superior when the detuning frequency is zero.

The derived closed-form transfer function allows us to calculate the small-signal behaviors and optimize the MRR PD design for different aims. The resonant wavelength is verified to be the optimal operating wavelength. The responsivity of PD can be further improved if the PAT effect can be enlarged.

The wavelength-selective photodetection makes the MRR PD promising in the WDM $\mathrm{Rx}$ application. There is no need for an additional optical filter and a small resonator size can enable a small footprint for the Rx. All-Si photodetection without Ge growth can also reduce material costs and simplify the process complexity. In addition, if the MRR PD can work at a higher bias voltage, such as an APD, the Rx sensitivity can be improved. Further work is required to check its performance as an APD, and a more complex model can be established to analyze the response for an MRR APD.

Author Contributions: Y.P. built the model, simulated the thermal effect, and wrote the manuscript. W.V.S. participated in the development of the model. S.C. participated in the measurements. Y.Y. designed and measured the devices. Y.Y., Z.H., M.F. and R.G.B. supervised the study. All the authors contributed to this paper. All authors have read and agreed to the published version of the manuscript.

Funding: This research received no external funding.

Data Availability Statement: The data that support the findings of this study are available from the authors upon reasonable request.

Conflicts of Interest: The authors declare no conflict of interest. 


\begin{abstract}
Appendix A
The NRZ and PAM4 eye diagrams of the MRR PD were measured with the pseudorandom bit sequence 9 (PRBS9) signals, which were generated with a 92 GSa/s arbitrary waveform generator (AWG). The optical signal from an O-band tunable laser was modulated by a $65 \mathrm{GHz}$ MZM. The signal was then amplified with a praseodymium-doped fiber amplifier (PDFA). The RF power from the all-Si MRR PD was detected after a bias tee by a $60 \mathrm{GHz}$ digital communication analyzer oscilloscope (DCA). The eye diagrams were measured at resonant wavelength at $-6.4 \mathrm{~V}$ without an equalizer and have been demonstrated in [35]. As shown in Figure A1, the MRR PD can support the NRZ signal with a data rate up to $80 \mathrm{~Gb} / \mathrm{s}$ and a PAM4 signal with a data rate up to $100 \mathrm{~Gb} / \mathrm{s}$. The eye opening at PAM4 modulation is mainly limited by the responsivity of PD.
\end{abstract}

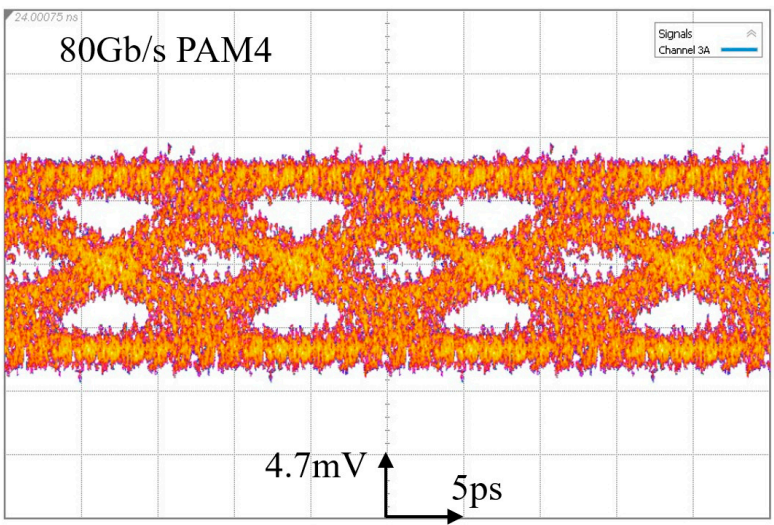

(a)

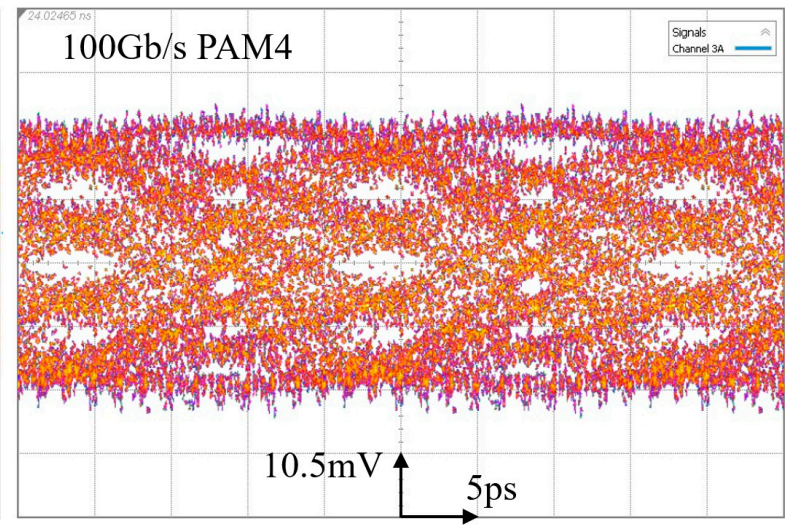

(b)

Figure A1. Measured eye diagrams with (a) $80 \mathrm{~Gb} / \mathrm{s}$ NRZ and (b) $100 \mathrm{~Gb} / \mathrm{s}$ PAM4 operations of a Si MRR PD.

\title{
Appendix B
}

To better understand the performance of our MRR PDs, the comparison of different allSi PDs/APDs is demonstrated in Table A1. Compared to other waveguide PDs, our MRR PDs achieved comparable bandwidth and high bit rate transmission with a compact size.

Table A1. Comparison of all-Si PDs and APDs.

\begin{tabular}{|c|c|c|c|c|}
\hline Device & Size & Responsivity & Bandwidth & Bit Rate \\
\hline This work & $12 \mu \mathrm{m}$ radius ring & $\begin{array}{c}0.1 \mathrm{~A} / \mathrm{W} \text { at }-6 \mathrm{~V} \\
(0.4 \mathrm{~A} / \mathrm{W} \text { at }-6.4 \mathrm{~V})\end{array}$ & $25 \mathrm{GHz}$ & $\begin{array}{c}80 \mathrm{~Gb} / \mathrm{s}(\mathrm{NRZ}) \\
100 \mathrm{~Gb} / \mathrm{s}(\mathrm{PAM} 4)\end{array}$ \\
\hline Mehta et al. [37] & $5 \mu \mathrm{m}$ radius ring & $0.52 \mathrm{~A} / \mathrm{W}$ & $5 \mathrm{GHz}$ & $12 \mathrm{~Gb} / \mathrm{s}$ \\
\hline Sakib et al. [12] & $10 \mu \mathrm{m}$ radius ring & $0.7 \mathrm{~A} / \mathrm{W}$ & $10 \mathrm{GHz}$ & $\begin{array}{c}56 \mathrm{~Gb} / \mathrm{s} \text { (NRZ) } \\
112 \mathrm{~Gb} / \mathrm{s} \text { (PAM4) }\end{array}$ \\
\hline Li et al. [11] & $12 \mu \mathrm{m}$ radius racetrack & $0.048 \mathrm{~A} / \mathrm{W}$ & $20 \mathrm{GHz}$ & $40 \mathrm{~Gb} / \mathrm{s}$ (NRZ) \\
\hline You et al. [10] & $600 \mu \mathrm{m}$ long waveguide & $10 \mathrm{~A} / \mathrm{W}$ & $10 \mathrm{GHz}$ & / \\
\hline Zhu et al. [38] & $3 \mathrm{~mm}$ long waveguide & $0.54 \mathrm{~A} / \mathrm{W}$ & $26 \mathrm{GHz}$ & $28 \mathrm{~Gb} / \mathrm{s}$ (NRZ) \\
\hline
\end{tabular}

\section{References}

1. Soref, R. The past, present, and future of silicon photonics. IEEE J. Sel. Top. Quantum Electron. 2006, 12, 1678-1687. [CrossRef]

2. Vivien, L.; Pavesi, L. (Eds.) Handbook of Silicon Photonics; Taylor \& Francis: Abingdon, UK, 2016.

3. Huang, Z.; Li, C.; Liang, D.; Yu, K.; Santori, C.; Fiorentino, M.; Sorin, W.; Palermo, S.; Beausoleil, R.G. 25 Gbps low-voltage waveguide Si-Ge avalanche photodiode. Optica 2016, 3, 793-798. [CrossRef]

4. Beling, A.; Cross, A.S.; Piels, M.; Peters, J.; Zhou, Q.; Bowers, J.E.; Campbell, J.C. InP-based waveguide photodiodes heterogeneously integrated on silicon-on-insulator for photonic microwave generation. Opt. Express 2013, 21, 25901-25906. [CrossRef] 
5. Tossoun, B.; Kurczveil, G.; Zhang, C.; Descos, A.; Huang, Z.; Beling, A.; Campbell, J.C.; Liang, D.; Beausoleil, R.G. Indium arsenide quantum dot waveguide photodiodes heterogeneously integrated on silicon. Optica 2019, 6, 1277-1281. [CrossRef]

6. Yuan, Y.; Jung, D.; Sun, K.; Zheng, J.; Jones, A.H.; Bowers, J.E.; Campbell, J.C. III-V on silicon avalanche photodiodes by heteroepitaxy. Opt. Lett. 2019, 44, 3538-3541. [CrossRef]

7. Ackert, J.J.; Karar, A.S.; Paez, D.J.; Jessop, P.E.; Cartledge, J.C.; Knights, A.P. 10 Gbps silicon waveguide-integrated infrared avalanche photodiode. Opt. Express 2013, 21, 19530-19537. [CrossRef]

8. Mailoa, J.P.; Akey, A.J.; Simmons, C.B.; Hutchinson, D.; Mathews, J.; Sullivan, J.T.; Recht, D.; Winkler, M.T.; Williams, J.S.; Warrender, J.M.; et al. Room-temperature sub-band gap optoelectronic response of hyperdoped silicon. Nat. Commun. 2014, 5, 3011. [CrossRef]

9. Li, Y.; Feng, S.; Zhang, Y.; Poon, A.W. Sub-bandgap linear-absorption-based photodetectors in avalanche mode in PN-diodeintegrated silicon microring resonators. Opt. Lett. 2013, 38, 5200-5203. [CrossRef]

10. You, J.B.; Kwon, H.; Kim, J.; Park, H.H.; Yu, K. Photon-assisted tunneling for sub-bandgap light detection in silicon PN-doped waveguides. Opt. Express 2017, 25, 4284-4297. [CrossRef]

11. Li, X.; Li, Z.; Xiao, X.; Xu, H.; Yu, J.; Yu, Y. 40 Gb/s all-silicon photodetector based on microring resonators. IEEE Photonics Technol. Lett. 2015, 27, 729-732. [CrossRef]

12. Sakib, M.; Liao, P.; Kumar, R.; Huang, D.; Su, G.L.; Ma, C.; Rong, H. A 112 Gb/s all-silicon micro-ring photodetector for datacom applications. In Optical Fiber Communication Conference; Optical Society of America: California, CA, USA, 8 March 2020; p. Th4A-2.

13. Karimelahi, S.; Sheikholeslami, A. Ring modulator small-signal response analysis based on pole-zero representation. Opt. Express 2016, 24, 7585-7599. [CrossRef]

14. Müller, J.; Merget, F.; Azadeh, S.S.; Hauck, J.; García, S.R.; Shen, B.; Witzens, J. Optical peaking enhancement in high-speed ring modulators. Sci. Rep. 2014, 4, 6310. [CrossRef]

15. Pile, B.; Taylor, G. Small-signal analysis of microring resonator modulators. Opt. Express 2014, 22, 14913-14928. [CrossRef]

16. Yuan, Y.; Sorin, W.V.; Huang, Z.; Liang, D.; Fiorentino, M.; Beausoleil, R.G. A 100 Gb/s PAM4 Two-Segment Silicon Microring Resonator Modulator. In Proceedings of the 2021 IEEE Photonics Conference (IPC), Vancouver, BC, Canada, 18-21 October 2021; pp. 1-2.

17. Song, J.; Eu-Jin, A.L.; Luo, X.; Huang, Y.; Tu, X.; Jia, L.; Fang, Q.; Liow, T.Y.; Yu, M.; Lo, G.Q. A microring resonator photodetector for enhancement in L-band performance. Opt. Express 2014, 22, 26976-26984. [CrossRef] [PubMed]

18. Hosseinifar, M.; Ahmadi, V.; Abaeiani, G. Design and optimization of high-performance microring-based uni-traveling carrier photodiode for WDM optical systems. Opt. Commun. 2012, 285, 2081-2084. [CrossRef]

19. Schuler, S.; Muench, J.E.; Ruocco, A.; Balci, O.; van Thourhout, D.; Sorianello, V.; Romagnoli, M.; Watanabe, K.; Taniguchi, T.; Goykhman, I.; et al. High-responsivity graphene photodetectors integrated on silicon microring resonators. Nat. Commun. 2021, 12, 3733. [CrossRef]

20. Ren, Y.; Van, V. Ultrawide-band silicon microring avalanche photodiode with linear photocurrent-wavelength response. Photonics Res. 2021, 9, 2303-2308. [CrossRef]

21. Padmaraju, K.; Bergman, K. Resolving the thermal challenges for silicon microring resonator devices. Nanophotonics 2014, 3 , 269-281. [CrossRef]

22. Huang, C.; Fan, J.; Zhu, L. Dynamic nonlinear thermal optical effects in coupled ring resonators. AIP Adv. 2012, 2, 032131. [CrossRef]

23. Xu, Q.; Lipson, M. Carrier-induced optical bistability in silicon ring resonators. Opt. Lett. 2006, 31, 341-343. [CrossRef]

24. Wu, S.L.; Wun, J.M.; Chao, R.L.; Huang, J.J.; Wang, N.W.; Jan, Y.H.; Chen, H.S.; Ni, C.J.; Chang, H.S.; Chou, E.; et al. High-speed In $0.52 \mathrm{Al} 0.48$ As based avalanche photodiode with top-illuminated design for $100 \mathrm{~Gb} / \mathrm{s}$ ER-4 system. J. Lightwave Technol. 2018, 36, 5505-5510. [CrossRef]

25. Peng, Y.; Sun, K.; Shen, Y.; Beling, A.; Campbell, J.C. High-Power and High-Linearity Photodiodes at 1064 nm. J. Lightwave Technol. 2020, 38, 4850-4856. [CrossRef]

26. Little, B.E.; Chu, S.T.; Haus, H.A.; Foresi, J.A.; Laine, J.P. Microring resonator channel dropping filters. J. Lightwave Technol. 1997, 15, 998-1005. [CrossRef]

27. Zhang, L.; Li, Y.; Yang, J.Y.; Song, M.; Beausoleil, R.G.; Willner, A.E. Silicon-based microring resonator modulators for intensity modulation. IEEE J. Sel. Top. Quantum Electron. 2009, 16, 149-158. [CrossRef]

28. Van, V. Optical Microring Resonators: Theory, Techniques, and Applications; CRC Press: Boca Raton, FL, USA, 2016.

29. Barclay, P.E.; Srinivasan, K.; Painter, O. Nonlinear response of silicon photonic crystal microresonators excited via an integrated waveguide and fiber taper. Opt. Express 2005, 13, 801-820. [CrossRef]

30. Isenberg, J.; Warta, W. Free carrier absorption in heavily doped silicon layers. Appl. Phys. Lett. 2004, 84, 2265-2267. [CrossRef]

31. Suzuki, N. FDTD analysis of two-photon absorption and free-carrier absorption in Si high-index-contrast waveguides. J. Lightwave Technol. 2007, 25, 2495-2501. [CrossRef]

32. Shen, Y.; Gaskins, J.; Xie, X.; Foley, B.M.; Cheaito, R.; Hopkins, P.E.; Campbell, J.C. Thermal analysis of high-power flip-chipbonded photodiodes. J. Lightwave Technol. 2017, 35, 4242-4246. [CrossRef]

33. Carmon, T.; Yang, L.; Vahala, K.J. Dynamical thermal behavior and thermal self-stability of microcavities. Opt. Express 2004, 12, 4742-4750. [CrossRef] 
34. Xu, D.X.; Delâge, A.; Verly, P.; Janz, S.; Wang, S.; Vachon, M.; Ma, P.; Lapointe, J.; Melati, D.; Cheben, P.; et al. Empirical model for the temperature dependence of silicon refractive index from $\mathrm{O}$ to $\mathrm{C}$ band based on waveguide measurements. Opt. Express 2019, 27, 27229-27241. [CrossRef]

35. Yuan, Y.; Sorin, W.V.; Cheung, S.; Peng, Y.; Huang, Z.; Fiorentino, M.; Beausoleil, R.G. A 4 × 100 Gb/s DWDM Optical Link with all-Silicon Microring Transmitters and Receivers. In Proceedings of the Asia Communications and Photonics Conference, Shanghai, China, 24-27 October 2021.

36. Dinu, M.; Quochi, F.; Garcia, H. Third-order nonlinearities in silicon at telecom wavelengths. Appl. Phys. Lett. 2003, 82, 2954-2956. [CrossRef]

37. Mehta, N.; Sun, C.; Wade, M.; Stojanović, V. A differential optical receiver with monolithic split-microring photodetector. IEEE J. Solid-State Circuits 2019, 54, 2230-2242. [CrossRef]

38. Zhu, H.; Goi, K.; Ogawa, K. All-Silicon waveguide photodetection for low-bias power monitoring and 20-km 28-Gb/s NRZ-OOK signal transmission. IEEE J. Sel. Top. Quantum Electron. 2017, 24, 1-7. [CrossRef] 